\title{
Existence of Multiple Solutions for $p$-Laplacian Problems Involving Critical Sobolev-Hardy Exponents and Singular Potential
}

\section{Zeid I. Almuhiameed}

Department of Mathematics, College of Science, Qassim University, Buraidah, KSA

Email: ksapr0066@yahoo.com

How to cite this paper: Almuhiameed, Z.I. (2020) Existence of Multiple Solution$\mathrm{s}$ for $p$-Laplacian Problems Involving Critical Sobolev-Hardy Exponents and Singular Potential. Open Access Library Journal, 7, e6812.

https://doi.org/10.4236/oalib.1106812

Received: September 11, 2020

Accepted: October 20, 2020

Published: October 23, 2020

Copyright (c) 2020 by author(s) and Scientific Research Publishing Inc.

This work is licensed under the Creative Commons Attribution International License $(\mathrm{CC}$ BY 4.0).

http://creativecommons.org/licenses/by/4.0/

\section{Abstract}

In this paper, we establish the existence of multiple solutions for $p$-Laplacian problems involving critical exponents and singular potential, by using Ekeland's variational principle and mountain pass theorem without Palais-Smale conditions.

\section{Subject Areas}

Mathematical Analysis

\section{Keywords}

p-Laplacian Problems, Ekeland's Variational Principle, Mountain Pass Theorem, Critical Hardy-Sobolev Exponent, Singular Potential

\section{Open Access}

\section{Introduction}

The aim of this paper is to establish the existence and multiplicity of solutions to the following quasilinear elliptic problem

$\left(\mathcal{P}_{\lambda, \mu}\right)\left\{\begin{array}{l}-\Delta_{p} u-\mu|x|^{-p}|u|^{p-2} u=|x|^{-s}|u|^{q-2} u+\lambda g(x) \text { in } \mathbb{R}^{N}, x \neq 0 \\ u \in \mathcal{D}_{1}^{p}\left(\mathbb{R}^{N}\right),\end{array}\right.$

where $\Delta_{p} u=\operatorname{div}\left(|\nabla u|^{p-2} \nabla u\right), 1<p<N, N$ is a integer, $x \in$ $\mathbb{R}^{N},-\infty<\mu<\bar{\mu}_{p}:=((N-p) / p)^{p}, 0 \leq s<p, q:=p^{*}(s)=$ $p(N-s) /(N-p)$ is the critical Sobolev-Hardy exponent, $\lambda$ and $\mu$ are positive parameters which we will specify later, and $g$ is a continuous function on $\mathbb{R}^{N}$.

Let $\mathcal{H}_{\mu}=\mathcal{D}_{1}^{p}\left(\mathbb{R}^{N}\right)$ be the space defined as the completion of $\mathcal{C}_{c}^{\infty}\left(\mathbb{R}^{N}\right)$ with respect to the norm

$$
\|\nabla u\|_{p}=\left(\int_{\mathcal{R}^{N}}|\nabla u|^{p} d x\right)^{\frac{1}{p}}
$$


When $\mu<\bar{\mu}_{p}$, Hardy type inequality implies that the norm

$$
\|u\|=\|u\|_{\mu, p}=\left(\int_{\mathcal{R}^{N}}\left(|\nabla u|^{p}-\mu|y|^{-p}|u|^{p}\right) d x\right)^{1 / p},
$$

is will defined in $\mathcal{H}_{\mu}$ and $\|$.$\| is equivalent to \|\nabla \cdot\|_{p}$; since the following inequalities hold:

$$
\begin{aligned}
& \left(1-\left(\max (\mu, 0) / \bar{\mu}_{p}\right)\right)^{1 / p}\|\nabla u\|_{p} \\
& \leq\|u\| \leq\left(1-\left(\min (\mu, 0) / \bar{\mu}_{p}\right)\right)^{1 / p}\|\nabla u\|_{p},
\end{aligned}
$$

for all $u \in \mathcal{H}_{\mu}$.

We define the weighted Sobolev space $\mathcal{D}:=\mathcal{H}_{\mu} \cap L^{p}\left(\mathbb{R}^{N},|x|^{-s} d x\right)$ which is a Banach space with respect to the norm defined by $\mathcal{N}(u):=$ $\|u\|_{\mu}+\left(\int_{\mathbb{R}^{N}}|x|^{-s}|u|^{q} d x\right)^{1 / q}$.

Several existence results are available in the case $p=2$, we quote for example [1,2], and the references therein. For more details, when $h \equiv$ $1, \mu=0$ and $q=2^{*}$, the regular problem $\left(\mathcal{P}_{1,0}\right)$ has been considered, on the bounded domain $\Omega$, by Tarantello [3]. She proved that for $g \in$ $\left(H_{0}^{1}(\Omega)\right)^{\prime}$ not identically zero and satisfying a suitable condition, the problem considered admits two solutions. Also, they are two nontrivial nonnegative solutions when $g$ is nonnegative.

Since our approach is variational, we define the functional $I_{\lambda, \mu}$ on $\mathcal{D}$ by

$$
I_{\lambda, \mu}(u):(1 / p)\|u\|^{p}-(1 / q) \int_{\mathbb{R}^{N}}|x|^{-s}|u|^{q} d x-\lambda \int_{\mathbb{R}^{N}} g(x) u d x .
$$

Throughout this work, we consider the following assumption

$$
g \in \mathcal{H}_{\mu}^{\prime}\left(\text { dual of } \mathcal{H}_{\mu}\right)
$$

In our work, we prove the existence of at least two distinct critical points of $I_{\lambda, \mu}$, one by the Ekeland variational principle in [4] with negative energy, and the other by mountain pass theorem in [5] without Palais-Smale conditions with positive energy.

Our main result is given as follows

Theorem 1. Suppose that $0 \leq s<2, \mu<\bar{\mu}_{p}$, hypothesis $(G)$ holds, $g \in \mathcal{H}_{\mu}^{\prime} \cap\left(\mathbb{R}^{N}\right)$ and $g \neq 0$. Then there exists $\Lambda_{*}>0$ such that the problem $\left(\mathcal{P}_{\lambda, \mu}\right)$ has at least two solutions for any $\lambda \in\left(0, \Lambda_{*}\right)$.

This paper is organized as follows. In Section 2, we give some preliminaries. Section 3 is devoted to the proof of Theorem 1.

\section{Preliminaries}

The first inequality that we need is the Hardy inequality

$$
\int_{\mathcal{R}^{N}}|\nabla u|^{p} d x \geq \bar{\mu}, p \int_{\mathcal{R}^{N}}|x|^{-p}|u|^{p} d x, \text { for all } u \in \mathcal{D}_{1}^{p}\left(\mathbb{R}^{N}\right),
$$

the constant $\bar{\mu}_{p}:=((N-p) / p)^{p}$ is sharp but not achieved [2].

Definition 1. An entire solution $v$ to $\left(\mathcal{P}_{\lambda, \mu}\right)$ is a ground state solution if it achieves the best constant

$$
S_{\mu, p}=S_{\mu, p}(N):=\inf _{v \in \mathcal{H}_{\mu}\left(\mathbb{R}^{N} \backslash 0\right)} \frac{\left(\int_{\mathcal{R}^{N}}\left(|\nabla u|^{p}-\mu|x|^{-p}|u|^{p}\right) d x\right) d x}{\left(\int_{\mathbb{R}^{N}}|x|^{-s}|v|^{q} d x\right)^{p / q}},
$$


Lemma 1. [6] Assume that $0 \leq s<2$ and $\mu<\bar{\mu}_{p}$. Then the infimum $S_{\mu, p}$ is achieved on $\mathcal{H}_{\mu}$.

Lemma 2. Let $\left(u_{n}\right) \subset \mathcal{D}$ be a Palais-Smale sequence [(PS)c in short] of $I_{\lambda, \mu}$, i.e.,

$$
I_{\lambda, \mu}\left(u_{n}\right)=c+o_{n}(1) \text { and } I_{\lambda, \mu}^{\prime}\left(u_{n}\right)=o_{n}(1),
$$

where $o_{n}(1)$ tends to 0 as $n$ goes at infinity, for $c \in \mathbb{R}$. Then, $u_{n} \rightarrow u$ in $\mathcal{D}$ and $I_{\lambda, \mu}^{\prime}(u)=0$.

Proof. From (2.3), we have

$(1 / p)\left\|u_{n}\right\|^{p}-(1 / q) \int_{\mathbb{R}^{N}}|x|^{-s}\left|u_{n}\right|^{q} d x-\lambda \int_{\mathbb{R}^{N}} g(x) u_{n} d x=c+o_{n}(1)$

and

$$
\left\|u_{n}\right\|^{p}-\int_{\mathbb{R}^{N}}|x|^{-s}\left|u_{n}\right|^{q} d x-\lambda \int_{\mathbb{R}^{N}} g(x) u_{n} d x=o_{n}(1), \text { for } n \text { large, }
$$

where $o_{n}(1)$ denotes $o_{n}(1) \longrightarrow 0$ as $n \longrightarrow \infty$. Then,

$$
\begin{aligned}
c+o_{n}(1) & =I_{\lambda, \mu}\left(u_{n}\right)-(1 / q)\left\langle I_{\lambda, \mu}^{\prime}\left(u_{n}\right), u_{n}\right\rangle \\
& \geq((q-p) / p q)\left\|u_{n}\right\|^{p}-\lambda((q-1) / q)\|g\|_{\mathcal{H}_{\mu}^{\prime}}\left\|u_{n}\right\|,
\end{aligned}
$$

$\left(u_{n}\right)$ is bounded in $\mathcal{D}$. Up to a subsequence if necessary, we obtain that

$$
\begin{aligned}
& u_{n} \rightarrow u \text { in } \mathcal{D} \\
& u_{n} \rightarrow u \text { in } L_{q}\left(\mathbb{R}^{N} ;|x|^{-s}\right) \\
& u_{n} \longrightarrow u \text { a.e in } \mathbb{R}^{N} .
\end{aligned}
$$

Consequently, we get

$$
I_{\lambda, \mu}^{\prime}(u)=0
$$

Lemma 3. Let $\left(u_{n}\right) \subset \mathcal{D}$ be a Palais-Smale sequence of $I_{\lambda, \mu}$, i.e., for $c \in \mathbb{R}$. Then, $u_{n} \rightarrow u$ in $\mathcal{D}$, and either

$$
u_{n} \longrightarrow u \text { or } I_{\lambda, \mu}(u)+((q-p) / p q)\left(S_{\mu, q}\right)^{q /(q-p)} .
$$

for all $p \in\left(2,2^{*}\right]$.

Proof. We know that $\left(u_{n}\right)$ is bounded in $\mathcal{D}$. Up to a subsequence if necessary, we have that

$$
\begin{aligned}
& u_{n} \rightarrow u \text { in } \mathcal{D} \\
& u_{n} \longrightarrow u \text { a.e in } \mathbb{R}^{N} .
\end{aligned}
$$

Denote $v_{n}=u_{n}-u$, then $v_{n} \rightarrow 0$. As in Brézis and Lieb [2], we have

$$
\left|v_{n}\right|_{q}^{p}=\left|u_{n}\right|_{q}^{p}-|u|_{q}^{p}
$$

and

$$
\lim _{n \longrightarrow \infty} \int_{\mathbb{R}^{N}}\left(|x|^{-s}\left|u_{n}\right|^{q}-|x|^{-s}\left|u_{n}-u\right|^{q}\right) d x=\int_{\mathbb{R}^{N}}|x|^{-s}|u|^{q} d x .
$$


On the other hand, we obtain

$$
\lim _{n \rightarrow \infty} \int_{\mathbb{R}^{N}}|x|^{-s}\left|v_{n}\right|^{q} d x=\lim _{n \rightarrow \infty} \int_{\mathbb{R}^{N}}|x|^{-s}\left|v_{n}\right|^{q} d x .
$$

Then, we get

$$
I_{\lambda, \mu}\left(u_{n}\right)=I_{\lambda, \mu}(u)+(1 / p)\left\|v_{n}\right\|^{p}-(1 / q) \int_{\mathbb{R}^{N}}|x|^{-s}\left|v_{n}\right|^{q}+o_{n}(1)
$$

and

$$
\left\langle I_{\lambda, \mu}^{\prime}\left(u_{n}\right), u_{n}\right\rangle=\left\|v_{n}\right\|^{p}-\int_{\mathbb{R}^{N}}|x|^{-b}\left|v_{n}\right|^{q}+o_{n}(1) .
$$

Then we can assume that

$$
\lim _{n \longrightarrow \infty}\left\|v_{n}\right\|^{p}=\lim _{n \longrightarrow \infty} \int_{\mathbb{R}^{N}}|x|^{-s}\left|v_{n}\right|^{q}=l \geq 0 .
$$

Assume $l>0$, we have by definition of $S_{\mu, q}$

$$
l \geq S_{\mu, q}(l)^{p / q}
$$

and so that

$$
l \geq\left(S_{\mu, q}\right)^{q /(q-p)} .
$$

Thus we get

$$
\begin{aligned}
c & =I_{\lambda, \mu}(u)+((q-p) / p q) l \\
& \geq I_{\lambda, \mu}(u)+((q-p) / p q)\left(S_{\mu, q}\right)^{q /(q-p)} .
\end{aligned}
$$

\section{Proof of Theorem 1}

The proof of Theorem 1 is given in two parts.

\subsection{Existence of a Local Minimizer}

We prove that there exists $\lambda_{*}>0$ such that for any $\lambda \in\left(0, \lambda_{*}\right), I_{\lambda, \mu}$ can achieve a local minimizer.

First, we establish the following result.

Proposition 1. Suppose that $0 \leq s<2, \mu<\bar{\mu}_{p}$, hypothesis $(G)$ holds, $g \in \mathcal{H}_{\mu}^{\prime} \cap\left(\mathbb{R}^{N}\right)$ and $g \neq 0$. Then there exist positive constants $\lambda_{*}, \varrho$ and $\delta$ such for all $\lambda \in\left(0, \lambda_{*}\right)$ we have

$$
I_{\lambda, \mu}(u) \geq \delta>0 \text { for }\|u\|_{\mu}=\varrho .
$$

Proof. By the Holder inequality and the definition of $S_{\mu, q}$, we get for all $u \in \mathcal{D} \backslash\{0\}$ and $\varepsilon>0$

$$
\begin{aligned}
& I_{\lambda, \mu}(u):=(1 / p)\|u\|^{p}-(1 / q) \int_{\mathbb{R}^{N}}|x|^{-s}|u|^{q} d x-\lambda \int_{\mathbb{R}^{N}} g(x) u d x, \\
& \geq(1 / p)\|u\|^{p}-(1 / q) S_{\mu, q}\|u\|^{q}-\lambda\|g\|_{\mathcal{H}_{\mu}^{\prime}}\|u\| \\
& \geq(1 / p-\varepsilon)\|u\|^{p}-(1 / p) S_{\mu, q}\|u\|^{q}-C_{\varepsilon}\|\lambda g\|_{\mathcal{H}_{\mu}^{\prime}} .
\end{aligned}
$$

Taking $\varepsilon<1 / p$ and $\varrho=\|u\|_{\mu}$, then there exist $\varrho>0$ small enough and a positive constant $\lambda_{*}$ such that

$$
I_{\lambda, \mu}(u) \geq \delta>0 \text { for }\|u\|_{\mu}=\varrho \text { and } \lambda \in\left(0, \lambda_{*}\right) .
$$


Since $g$ is a continuous function on $\mathbb{R}^{N}$, not identically zero, we can choose $\phi \in C_{0}^{\infty}\left(\mathbb{R}^{N} \backslash\{0\}\right)$ such that $\int_{\mathbb{R}^{N}} g(x) \phi d x>0$. It follows that for $t>0$ small,

$$
I_{\lambda, \mu}(t \phi):=\left(t^{p} / p\right)\|\phi\|^{p}-\left(t^{q} / q\right) \int_{\mathbb{R}^{N}}|x|^{-s}|\phi|^{q} d x-\lambda t \int_{\mathbb{R}^{N}} g(x) \phi d x<0 .
$$

We also assume that $t$ is so small enough such that $\|t \phi\|_{\mu}<\varrho$. Thus, we have

$$
c_{1}=\inf \left\{I_{\lambda, \mu}(u): u \in B_{\varrho}\right\}<0, \text { where } B_{\varrho}=\{u \in \mathcal{D}, \mathcal{N}(u) \leq \varrho\} .
$$

Using the Ekeland's variational principle, for the complete metric space $\bar{B}_{\varrho}$ with respect to the norm of $\mathcal{D}$, we can prove that there exists a $(P C)_{c_{1}}$ sequence $\left(u_{n}\right) \subset \bar{B}_{\varrho}$ such that $u_{n} \rightarrow u_{1}$ for some $u_{1}$ with $\mathcal{N}\left(u_{1}\right) \leq \varrho$.

Now, we claim that $u_{n} \longrightarrow u_{1}$. If not, by Lemma ??, we have

$$
\begin{aligned}
c_{1} & \geq I_{\lambda, \mu}\left(u_{1}\right)+((q-p) / p q)\left(S_{\mu, q}\right)^{q /(q-p)} \\
& \geq c_{1}+((q-p) / p q)\left(S_{\mu, q}\right)^{q /(q-p)} \\
& >c_{1}
\end{aligned}
$$

which is a contradiction.

Then we obtain a critical point $u_{1}$ of $I_{\lambda, \mu}$ for all $\lambda \in\left(0, \lambda_{*}\right)$ satisfying

$$
c_{1}=I_{\lambda, \mu}\left(u_{1}\right)<0 .
$$

On the other hand we have

$$
\begin{aligned}
c_{1} & =((q-p) / p q)\left\|u_{1}\right\|^{p}-((q-1) / q) \int_{\mathbb{R}^{N}} \lambda g(x) u_{1} d x \\
& \geq-(1 / p q)(q-1)^{p}(q-p)^{-1} \lambda^{p}\|g\|_{\mathcal{H}_{\mu}^{\prime}}^{p} .
\end{aligned}
$$

Thus $u_{1}$ is a nontrivial solution of our problem with negative energy.

\subsection{Existence of Mountain Pass Type Solution}

We use the mountain pass theorem without Palais-Smale conditions to prove the existence of a nontrivial solution with positive energy. For this, we need the following Lemma.

Lemma 4. Let $\lambda^{*}>0$ such that

$$
m_{\lambda, p}^{*}>0 \text { for all } \lambda \in\left(0, \lambda^{*}\right) .
$$

Then, there exist $\Lambda \in\left(0, \lambda^{*}\right)$ and $\varphi_{\varepsilon}(x) \in \mathcal{D}$ for $\varepsilon>0$ such

$$
\sup _{t \geq 0} I_{\lambda, \mu}\left(t \varphi_{\varepsilon}\right)<m_{\lambda, p}^{*}, \text { for all } \lambda \in(0, \Lambda) .
$$

Proof. Let

$$
\varphi_{\varepsilon}(x)=\left\{\begin{array}{ll}
\omega_{\varepsilon}(x) & \text { if } g(x) \geq 0 \text { for all } x \in \mathbb{R}^{N} \\
\omega_{\varepsilon}\left(x-x_{0}\right) & \text { if } g\left(x_{0}\right)>0 \text { for } x_{0} \in \mathbb{R}^{N} \\
-\omega_{\varepsilon}(x) & \text { if } g(x) \leq 0 \text { for all } x \in \mathbb{R}^{N}
\end{array},\right.
$$

where $\omega_{\varepsilon}$ verifies $(2.2)$. 
Then, we claim that there is an $\varepsilon_{0}$ such that

$$
\int_{\mathbb{R}^{N}} g(x) \varphi_{\varepsilon}(x)>0, \text { for any } \varepsilon \in\left(0, \varepsilon_{0}\right) .
$$

In fact, $g(x) \geq 0$ or $g(x) \leq 0$ for all $x \in \mathbb{R}^{N}$, and (3.6) holds obviously. If there exists an $x_{0} \in \mathbb{R}^{N}$ such that $g\left(x_{0}\right)>0$, by the continuity of $g(x)$ there is an $\eta>0$ such that $g(x)>0$ for all $x \in$ $B_{\eta}\left(x_{0}\right)$. Then, by the definition of $\omega_{\varepsilon}\left(x-x_{0}\right)$, it is easy to see that there exists an $\varepsilon_{0}$ small enough such that

$$
\int_{\mathbb{R}^{N}} g(x) \omega_{\varepsilon}\left(x-x_{0}\right)>0, \text { for any } \varepsilon \in\left(0, \varepsilon_{0}\right) .
$$

Now, we consider the following functions

$$
f(t)=I_{\lambda, \mu}\left(t \varphi_{\varepsilon}\right)
$$

and

$$
\tilde{f}(t)=\left(t^{p} / p\right)\left\|\varphi_{\varepsilon}(x)\right\|^{p}-\left(t^{q} / q\right) \int_{\mathbb{R}^{N}}|x|^{-s}\left|\varphi_{\varepsilon}(x)\right|^{q} d x .
$$

Then, we get for all $\lambda \in\left(0, \lambda^{*}\right)$

$$
0=f(0)<m_{\lambda, p}^{*} .
$$

By the continuity of $f(t)$, there exists $t_{1}$ a sufficiently small positive quantity such that

$$
f(t)<m_{\lambda, p}^{*},
$$

for all $t \in\left(0, t_{1}\right)$. On the other hand, we have

$$
\max _{t \geq 0} \tilde{f}(t)=((q-p) / p q)\left(S_{\mu, q}\right)^{q /(q-p)},
$$

then, we obtain

$$
\sup _{t \geq 0} I_{\lambda, \mu}\left(t \varphi_{\varepsilon}\right)<((q-p) / p q)\left(S_{\mu, q}\right)^{q /(q-p)}-\lambda t_{1} \int_{\mathbb{R}^{N}}|x|^{-s} g(x) \varphi_{\varepsilon} d x \text {. }
$$

Taking $\lambda>0$ such that

$$
\lambda t_{1} \int_{\mathbb{R}^{N}} g(x) \varphi_{\varepsilon} d x>(1 / p q)(q-1)(q-p)^{-1 / p} \lambda^{p}\|g\|_{\mathcal{H}_{\mu}^{\prime}}^{p} .
$$

By (3.6), we get

$$
0<\lambda<W
$$

where

$$
W:=\left(p q(q-p)^{1 / p}(q-1)^{-1}\right) t_{1}\left(\int_{\mathbb{R}^{N}} g(x) \varphi_{\varepsilon} d x\right)\|g\|_{\mathcal{H}_{\mu}^{\prime}}^{-p} .
$$

Set

$$
\Lambda=\min \left\{\lambda^{*}, W\right\}
$$

We deduce that

$$
\sup _{t \geq 0} I_{\lambda, \mu}\left(t \varphi_{\varepsilon}\right)<m_{\lambda, p}^{*}, \text { forall } \lambda \in(0, \Lambda) .
$$

Since $\lim _{t \rightarrow \infty} I_{\lambda, \mu}\left(t \varphi_{\varepsilon}\right)=-\infty$, we can choose $T>0$ large enough such that $I_{\lambda, \mu}\left(T \varphi_{\varepsilon}\right)<0$. From Proposition 1 , we have $I_{\lambda, \mu \mid \partial B_{\varrho}} \geq \delta>0$ 
for all $\lambda \in\left(0, \lambda_{*}\right)$. By mountain pass theorem without the PalaisSmale condition, there exists a $(P C)_{c_{2}}$ sequence $\left(u_{n}\right)$ in $\mathcal{D}$ which is characterized by

$$
c_{2}=\inf _{\gamma \in \Gamma} \max _{t \in[0,1]} I_{\lambda, \mu}(\gamma(t)),
$$

with

$$
\Gamma=\left\{\gamma \in C([0,1], \mathcal{D}), \gamma(0)=0, \gamma(1)=T \varphi_{\varepsilon}\right\} .
$$

Then, $\left(u_{n}\right)$ has a subsequence, still denoted by $\left(u_{n}\right)$ such that $u_{n} \rightarrow$ $u_{2}$ in $\mathcal{D}$. By Lemma 3 , if $u_{n}$ doesn't converge to $u_{2}$, we get

$$
c_{2} \geq I_{\lambda, \mu}\left(u_{2}\right)+((q-p) / p q)\left(S_{\mu, q}\right)^{q /(q-p)} \geq m_{\lambda, p}^{*},
$$

what contradicts the fact that, by Lemma 4 , we have

$$
\sup _{t \geq 0} I_{\lambda, \mu}\left(t \varphi_{\varepsilon}\right)<m_{\lambda, p}^{*}
$$

for all $\lambda \in(0, \Lambda)$. Then

$$
u_{n} \longrightarrow u_{2} \text { in } \mathcal{D} \text {. }
$$

Thus, we obtain a critical point $u_{2}$ of $I_{\lambda, \mu}$ for all $\lambda \in\left(0, \lambda_{*}\right)$ with

$$
\Lambda_{*}:=\min \left\{\lambda_{*}, \Lambda\right\}
$$

satisfying

$$
I_{\lambda, \mu}\left(u_{2}\right)>0
$$

\section{Conclusion}

In this work, we have searched the critical points as the minimizers of the energy functional associated to the problem. Under some sufficient conditions on coefficients of equation of (1.1) we have proved the existence of at least two distinct critical points of $I_{\lambda, \mu}$, one by the Ekeland variational principle with negative energy, and the other by mountain pass theorem without Palais-Smale conditions with positive energy.

\section{Acknowledgements}

The author gratefully acknowledge researchers collaborating in the mathematics department, on the effort made in this field under the number (1066) during the academic year 1442AH/2020-2021AD.

\section{Conflicts of Interest}

The author declares no conflicts of interest regarding the publication of this paper.

\section{References}

[1] Badiale, M. and Tarantello, G. (2002) A Sobolev-Hardy Inequality with Applications to a Nonlinear Elliptic Equation Arising in Astrophysics. Archive for Rational Mechanics and Analysis, 163, 252-293. https://doi.org/10.1007/s002050200201 
[2] Brézis, H. and Lieb, E. (1983) A Relation Between Point Convergence of Functions and Convergence of Functional. Proceedings of the American Mathematical Society, 88, 486-490.

https://doi.org/10.1090/S0002-9939-1983-0699419-3

[3] Tarantello, G. (1992) On Nonhomogeneous Elliptic Equations Involving Critical Sobolev Exponent. Annales de linstitut Henri Poincaré, 9, 281-304.

https://doi.org/10.1016/S0294-1449(16)30238-4

[4] Gazzini, M. and Mussina, R. (2009) On a Sobolev Type Inequality Related to the Weighted p-Laplace Operator. Journal of Mathematical Analysis and Applications, 352, 99-111.

[5] Badiale, M., Bergio, V. and Rolando, S. (2007) A Nonlinear Elliptic Equation with Singular Potential and Applications to Nonlinear Field Equations. Journal of the European Mathematical Society, 9, 355-381.

[6] Musina, R. (2008) Ground State Solution of a Critical Problem Involving Cylindrical Weights. Nonlinear Analysis, 68, 39723986 . 